\title{
PRP Injection Effect on Palatal Fistula Repair: A Comparative Study
}

\author{
DAWLAT EMARA, M.D.; KHALED MAKEEN, M.D.; MAMDOUH ABOULHASSAN, M.D. and \\ MARWAN A. ADEL, M.Sc. \\ The Department of Plastic \& Reconstructive Surgery, Faculty of Medicine, Cairo University
}

\begin{abstract}
Background: Palatal fistula after primary palate repair is an annoying problem for both patients and plastic surgeons. The incidence of fistula depends on the severity of the primary defect, the technique used for repair and the tension applied over the wound. Different methods are described for palatal fistula repair, starting from local mucoperiosteal flaps to free flaps.

The recurrence rate of fistula after primary repair is about $23 \%$, on the other hand the recurrence rate of fistula after its repair is higher as $25-33 \%$ up to $100 \%$. Fistulas are classified according to its site and size. PRP is an autologous blood product, it promotes healing of wounds by its high platelet concentration and multiple growth factors, and also it decreases the risk of infection and increases neovascularization. PRP is used in several clinical applications as in oral maxillofacial, cardiac and plastic surgery.
\end{abstract}

Methods: A comparative study was conducted on 20 patients with posterior palatal fistulas, who were divided into two groups, group A in which PRP was injected with fistula repair and group B fistula was repaired without PRP injection. Two flap palatoplasty ( 9 cases in each group) and Furlow technique (one case in each group) were used for both groups.

Results: A total of 16 patients were followed-up for recurrence rate of fistulas, statistical analysis showed that in group A $25 \%$ had a recurrence, while in group B 50\% of cases showed recurrence.

Conclusion: PRP injection with the surgical repair of the palatal fistula has a role in decreasing the recurrence rate of the fistula and decreasing the need for further surgical interventions also, it is an easy, safe procedure and at an economic price.

Key Words: Palatal fistula - PRP.

\section{INTRODUCTION}

Several techniques have been described to repair primary cleft palate. Experienced surgeons have good outcome, still they face complications associated with cleft palate surgery. To reach the optimum surgical outcome restoring palatal anatomy and function, closure in multiple layers to separate the nasal and oral lining and a tension free closure is the goal [1].
Fistulas are one of the annoying and that may be common complications following palate repair, they develop due to wound break down caused by undesirable wound tension leading to tissue ischemia, hematoma formation between layers of repair, infection, trauma to the flap, poor surgical technique and also due to the severity of the cleft palate type [2].

Pittsburgh introduced a classification system which is simple and anatomically based. It includes seven types "fistula at the uvula or bifid uvula type I, in soft palate is type II, at the junction of soft and hard palate is type III, in the hard palate is type IV, at the incisive foramen is type V, type VI is lingual-alveolar and type VII are labial-alveolar" Others described fistulas as intentional (resulting from unrepaired cleft) or unintentional (due to wound dehiscence causes) [2].

Fistulas are treated according to functionality as symptomatic (functional) fistula as the patient complains of regurgitation of fluid and food, impaired speech and VPI, or asymptomatic (non functional) [3].

Apparently it's difficult to repair fistulas due to scarring and limited mobility of tissues; different surgical and non surgical techniques were introduced to manage fistulas. The surgical method used for fistula repair depends on its site, size, previous surgical technique used for palate repair, the amount of scarring and availability of palatal and surrounding tissues [4].

PRP is used in plastic surgery. Many benefits were suggested as it promotes wound healing by regenerating epithelial cells, and increasing angiogenesis. In addition it's easily prepared, safe for the patients and being autologous has low rate of infection [5]. 
Gonzalez and Jimenez used PRP during closure of recurrent palatal fistulas as they claimed it reduced the recurrence rate of fistulas [6], the aim of this work is to study and evaluate the effect of PRP during palatal fistula repair and if it is helpful in decreasing the rate of recurrence of the fistulas.

\section{PATIENTS AND METHODS}

20 patients with posterior palatal fistulas were included in this comparative study; this was done in the pediatric hospital Cairo University between September 2016 and September 2017 with followup by photos after 3 and 6 months postoperative.

All patients were non syndromic cleft palate patients with posterior palatal fistulas less than 1 $\mathrm{cm}$ and their age ranged between 2 to 6 years with an average of 4 years, with primary palate repair that was done more than one year.

Detailed history and examination were done to all patients including evaluation of the velopharyngeal function using nasopharyngeoscope.

Preoperative laboratory investigations in the form of:

- Complete blood picture.

- Bleeding profile (PT,PTT and INR).

- Palate swab for culture and sensitivity.

Digital photography (intraoperative, and postoperative after 3 and 6 months) were taken.

The surgical techniques used for repair of posterior palatal fistula were either two flap palatoplasty ( 9 patients in each group) or Furlow palatoplasty (one patient in each group).

The patients were divided into two groups, group A were PRP was injected after the palatal fistula repair in the edge of the wound between the nasal and oral layer and group B without PRP injection with palatal fistula repair.

\section{Operative details:}

Patients were operated under general anesthesia in the supine position with head tilting 20 degrees for better vision. A systemic antibiotic was given to the patient. Mouth Gag (Fig. 1) was applied as the first step at the operating theater after induction of anesthesia to measure the size of the fistula using a caliber and pre-operative photos to be taken.

According to the preoperative plan, if the patient has VPI, Double opposing Z-plasty (Furlow) procedure was done. If no VPI, then two flap palato- plasty was done. If a concomitant anterior fistula was present, a buccal mucosal flap or a buccal fat flap was used to repair the anterior fistula.

Marking (Fig. 2) of the flaps and the site of the pedicle (the greater palatine artery) was done using sterile methylene blue before injection of $1 / 200$ 000 saline adrenaline and lidocaine.

Under sterile technique $10 \mathrm{cc}$ of peripheral venous blood was aspirated from the patient using the vacuette blood aspiration kit for the PRP preparation at this point in a container that has sodium citrate $3.2 \%$. The sample was immediately centrifuged using a tabletop centrifuge machine for 10 minutes at a speed of $1000 \mathrm{rpm}$ in the operative room temperature.

Then the tube is left to suspend for 5 minutes. Then the upper part of the centrifuged blood is transferred to another tube, and discarding the red blood cells. The new tube is then re-inserted in the centrifuge for 5 minutes at a speed of $2500 \mathrm{rpm}$.

The blood was separated into three layers based on density, the bottom layer containing the RBCs, middle layer containing the PRP consisting of platelets and WBCs (buffy coat), and the top layer containing platelet poor plasma (Fig. 3). The pellet is then aspirated in a syringe with $1 \mathrm{cc}$ plasma. The PRP mixture is left aside till the end of the procedure to be injected along the edge of the repaired wound (Fig. 4).

Surgery:

Incisions along the markings were done. Removal of any fibrous scar tissue from the primary repair was done. Exploration of the pedicle of the flaps and identification of the greater palatine artery was an essential step. Viability of the flaps was checked according to its color and bleeding from its edges.

According to the method of repair:

A- In cases of two flap palatoplasty:

Closure was done in an interrupted manner using absorbable Coated VICRYL sutures 5/0 along the nasal layer. Lateral relaxing incisions were done to decrease the tension along the midline sutures.

\section{B- In cases of Double Opposing Z-plasty (Furlow) repair:}

The oral flap is posteriorly based on the left side; it is raised with the levator muscle, the right sided flap above the muscle. The reverse pattern is planned for the oral side. The nasal flaps are 
transposed and the anterior oral mucosa closed. The closure was done as well using absorbable Coated VICRYL sutures. The use of relaxing incisions was done when necessary.

After closure, the PRP was injected, 5mm away from the wound edge using a $27 \mathrm{G}$ about $0.10 \mathrm{ml}$ $0.15 \mathrm{ml}$ was injected at each site between the nasal and oral layers. Spacing between each injection site was $1 \mathrm{~cm}$.

Applying Soft arm restraints that prevent flexion at the elbow at the end of the procedure, and it was kept for two weeks.

All patients were admitted to the in-patient section for 24 hours for close monitoring and detecting the early postoperative complications as hemorrhage \& breathing problems.

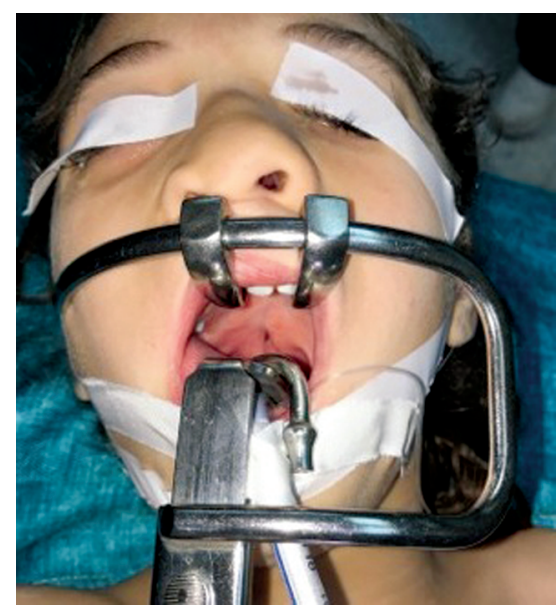

Fig. (1): Applying mouth gag.

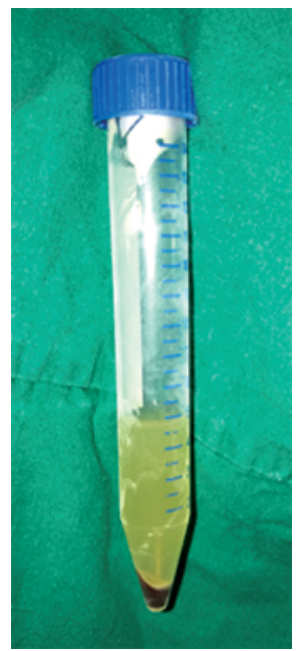

Fig. (3): After $2^{\text {nd }}$ centrifuge.
Patients were given oral antibiotics, pain killers as paracetamol, oral antifungal and decongestant nasal drops.

Post-operative feeding instructions as follows:

a- Drinking of clear fluids was encouraged to avoid oral fungation. The first five days post-operative only water and clear fluids were allowed.

b- The following five days milk was added to the previous regimen.

c- And the following five days soft diet was introduced, by using a syringe. Breast feeding \& bottle feeding were all prohibited for two weeks after the operation.

Follow-up by photography after the first week post-operative, and after 3 and 6 months postoperative.

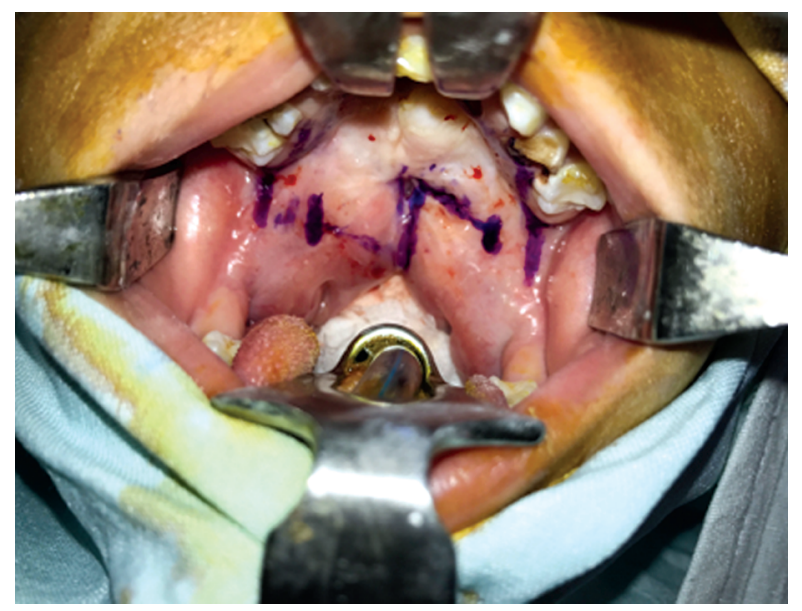

Fig. (2): Marking.

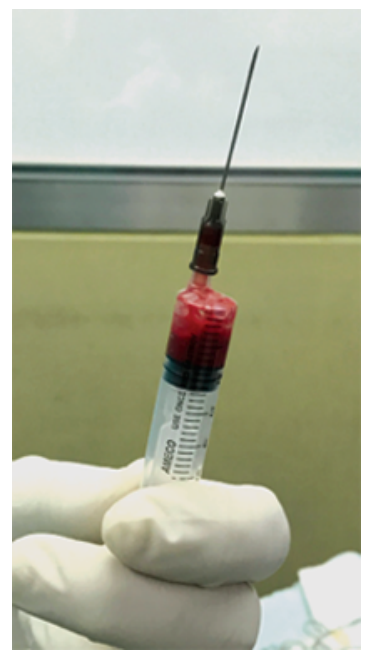

Fig. (4): PRP prepared for injection 


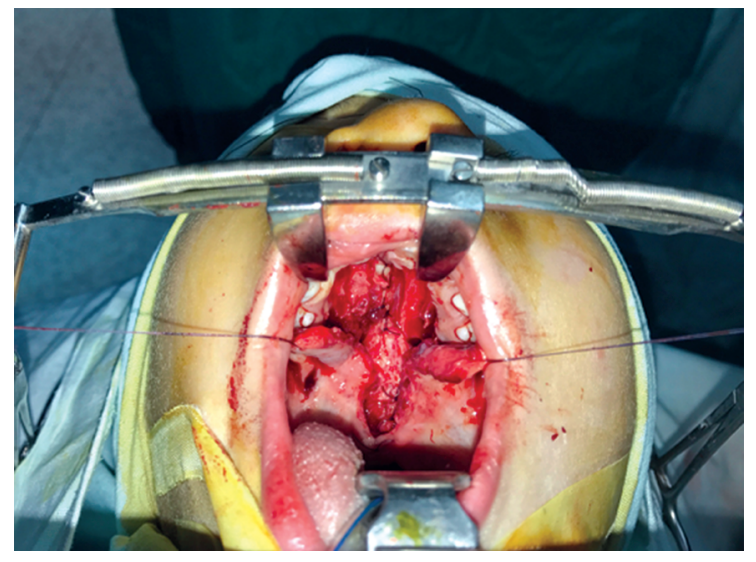

Fig. (5): Nasal layer closure.

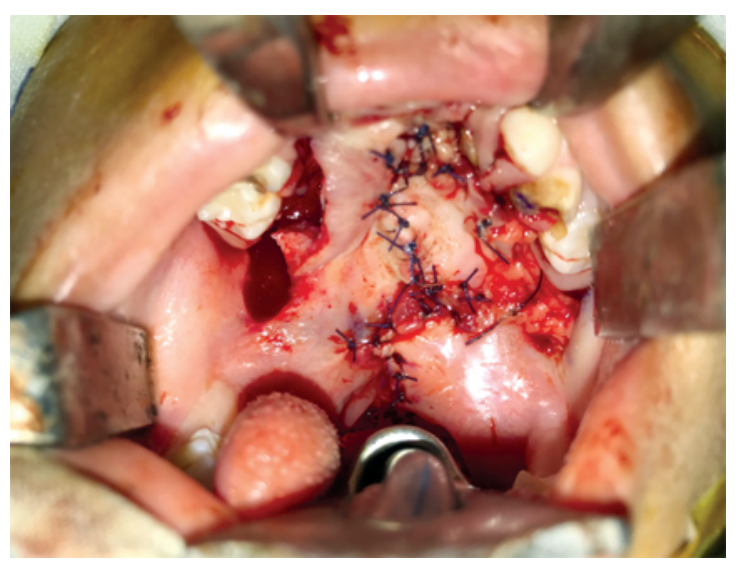

Fig. (7): Closure after Furlow technique.

\section{RESULTS}

Twenty cases were successfully operated upon in this study and they were 13 male patients and 7 females, with a fistulas size ranged from $0.6 \mathrm{~cm}$ to $1 \mathrm{~cm}$, the site of the fistulas according to Pittsburgh fistula classification system showed that the patients were of type 3 .

Two methods of repair were used, 18 patients were repaired by two flap palatoplasty technique and the other two patients were done by Furlow technique.

\section{They were divided into two groups:}

Group A included 10 patients were PRP was injected with fistula repair, 8 patients continued their follow-up and two patients had recurrence.

Group B included 10 patients with fistula repair without PRP injection, 8 patients continued followup and four patients had recurrence.

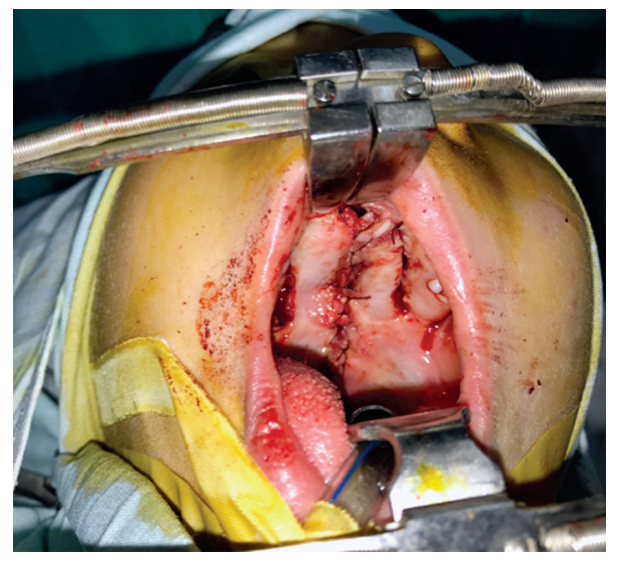

Fig. (6): Oral layer closure.

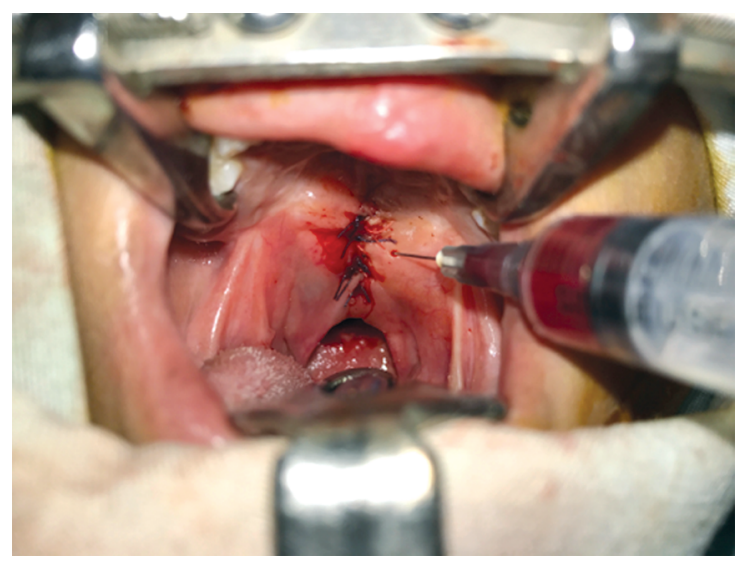

Fig. (8): Injection of PRP after closure.

Follow-up photos were taken for both groups: Intraoperative, 3 and 6 months postoperative.

A total of 16 patients continued their followup with a recurrence rate of $25 \%$ in group A and $50 \%$ recurrence rate in group B according to statistical results and $p$-value is (0.0508).

Table (1): Count and percentage of recurrence rates of palatal fistula.

\begin{tabular}{|c|c|c|c|}
\hline & \multicolumn{2}{|c|}{ Group } & \multirow{2}{*}{ Total } \\
\hline & PRP & Non-PRP & \\
\hline \multicolumn{4}{|l|}{ Recurrence: } \\
\hline \multicolumn{4}{|l|}{ No: } \\
\hline Count & 6 & 4 & 10 \\
\hline$\%$ Within group & $75 \%$ & $50 \%$ & $62.5 \%$ \\
\hline \multicolumn{4}{|l|}{ Yes: } \\
\hline Count & 2 & 4 & \\
\hline$\%$ Within group & $25 \%$ & $50 \%$ & $37.5 \%$ \\
\hline \multicolumn{4}{|l|}{ Total: } \\
\hline Count & 8 & 8 & 16 \\
\hline$\%$ Within group & $100 \%$ & $100 \%$ & $100 \%$ \\
\hline
\end{tabular}




\section{Case 1 (study group):}

A 5 years old female patient who presented with an anterior and posterior palatal fistula

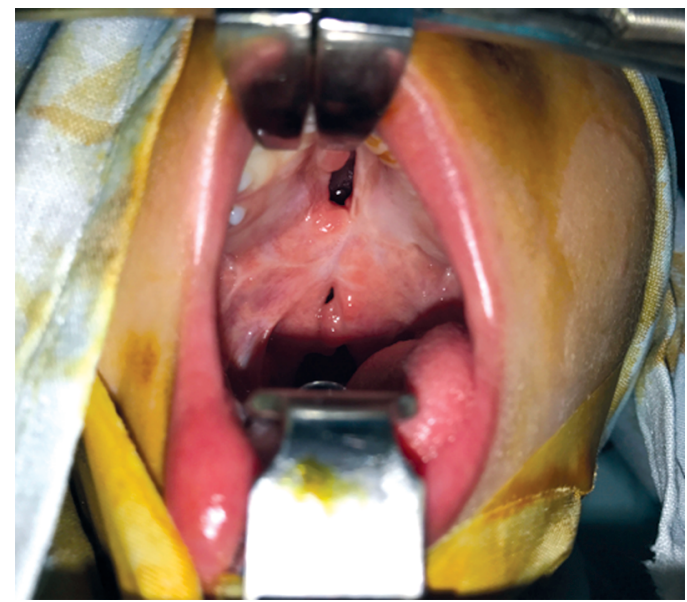

Fig. (9): Preoperative showing the fistula.

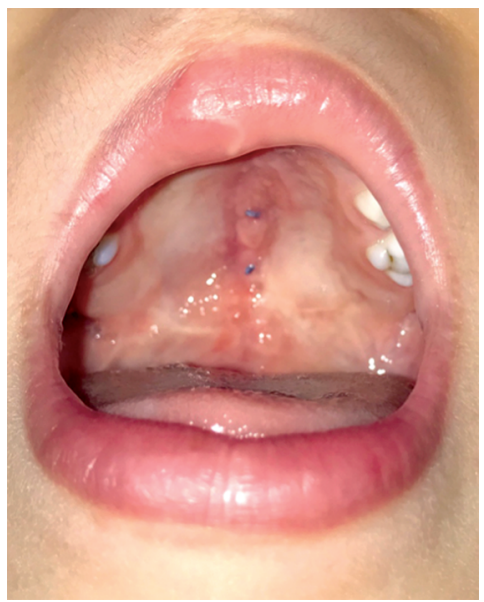

Fig. (11): 3 months post-operative.

Case 2 (study group):

A 3.5 years old male patient presented with posterior palatal fistula. (PSFC: Type III).

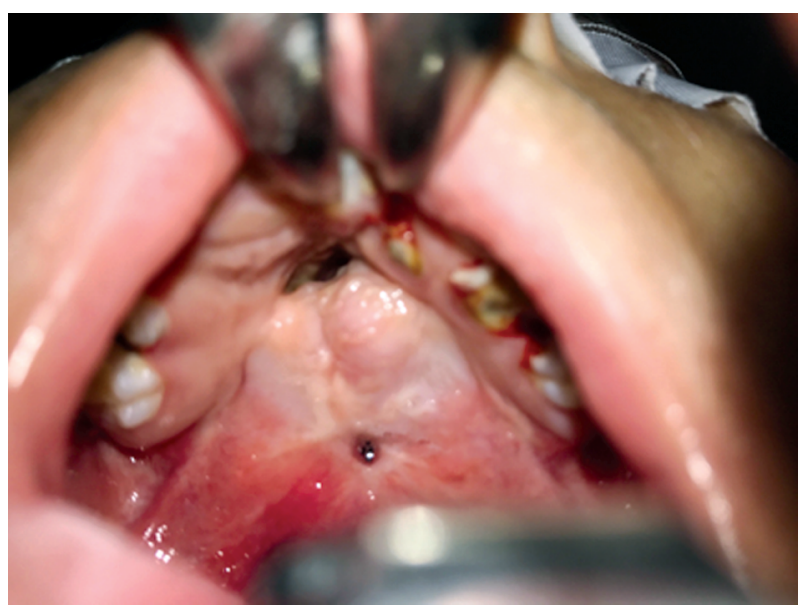

Fig. (13): Preoperative.
(PSFC: Type III). The size of the posterior fistula was $0.6 \mathrm{~mm}$. PRP injection at the end was done.

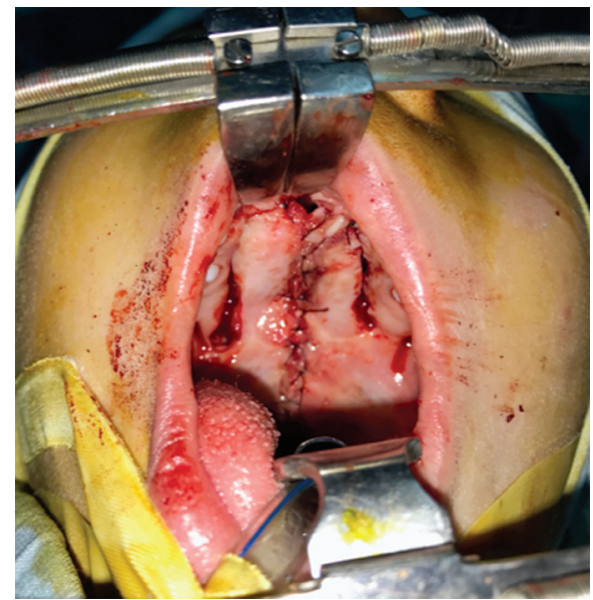

Fig. (10): Intra-operative, 2 flap palatoplasty repair.

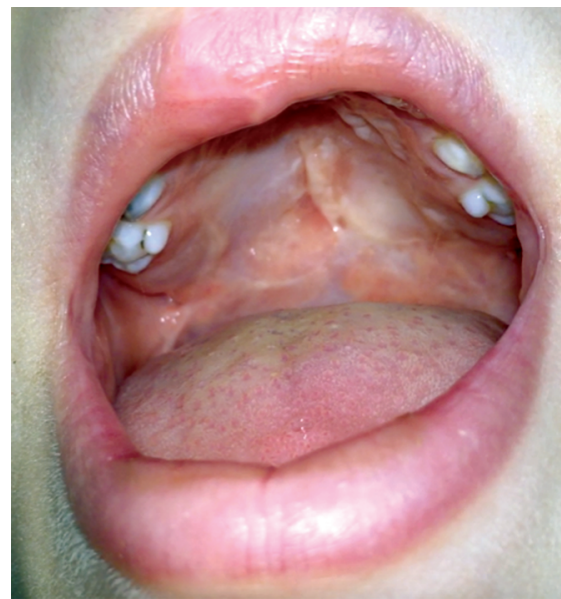

Fig. (12): 6 months post operative.

The size of the posterior fistula was $0.6 \mathrm{~mm}$. PRP injection at the end was done.

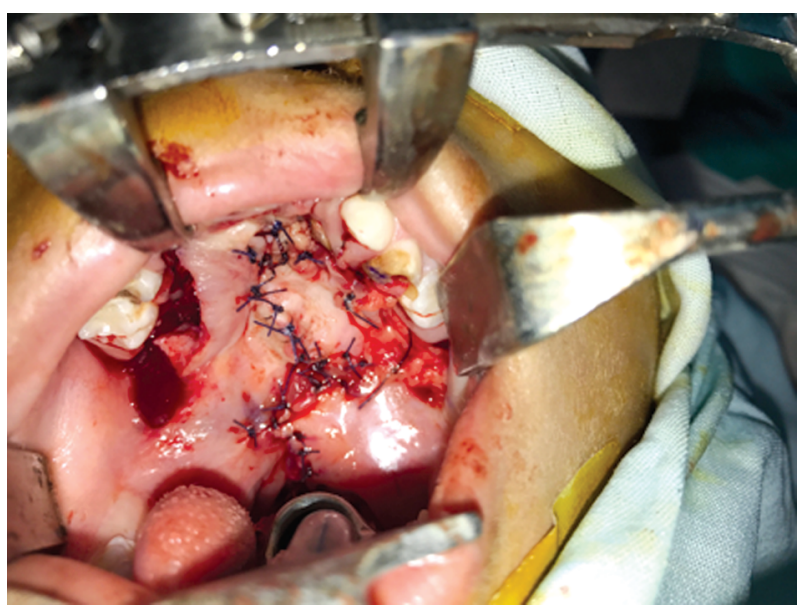

Fig. (14): Intraoperative using Furlow technique. 


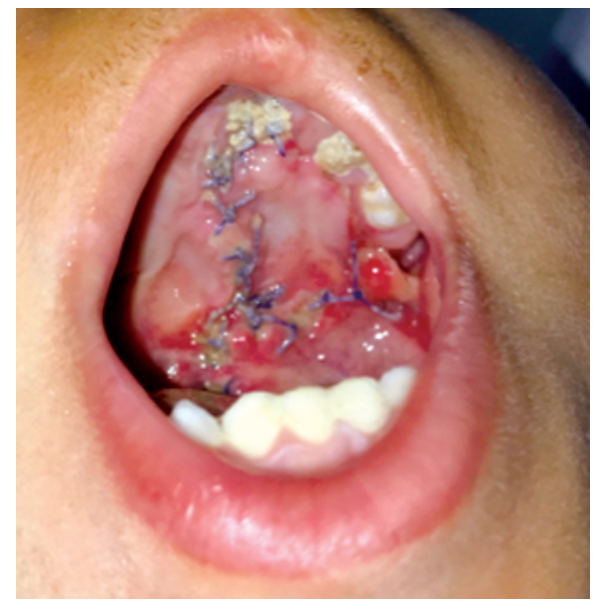

Fig. (15): One month postoperative.

\section{DISCUSSION}

Cleft palate repair is a tough operation due to a number of factors: Paucity of local tissues, young age, and the importance of an experienced surgeon. The most common and challenging complication following cleft palate repair is palatal fistula, with a high incidence of fistula after primary palatoplasty and even higher incidence of re-recurrence after repair of the palatal fistula. Severity of the fistula is related to: The type and width of the cleft palate, the technique used for the primary palatoplasty repair \& the tension over the wound, moreover persistent postoperative infection leads to oronasal fistulae [4].

Diah et al., described an algorithm for oronasal fistulae. After assessment of the patients, they divided them into two groups: Non-symptomatic patients and symptomatic patients (with inadequate velopharyngeal function). Symptomatic patients were examined using a nasopharyngoscope or a videofluroscope and performing the fistula occlusion test. Non-symptomatic patients were treated conservatively, while symptomatic patients were treated surgically [7].

Most common complaints of patients with symptomatic fistulae are leakage of fluids or food, hypernasality and regurgitation due to both the fistula and the velopharyngeal insufficiency. Those kinds of fistulae require surgical management [3].

Different techniques have been used for repair of different kinds of palatal fistulae such as local flaps, regional flaps or free tissue transfer. The choice of which technique to be used depends on the site and size of the fistula and availability of local tissues [4].

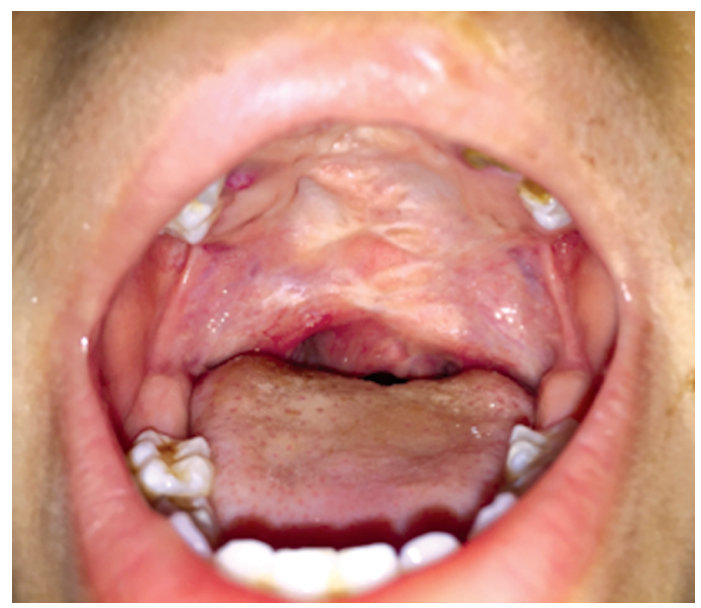

Fig. (16): 6 months postoperative

The goal of fistula correction is not only closure of the defect, but also improving the velopharyngeal function during speech. So, it is recommended to perform a re-operation using a two flap palatoplasty or double opposing z-plasty (Furlows technique) to close the fistula, lengthening the soft palate and reducing the wound tension [8].

We carried out a comparative study that included all symptomatic patients with a history of cleft palate that had been repaired, presented with posterior palatal fistula for at least one year, who attended the outpatient clinic during the period between September 2016 and September 2017.

In this study, the patients were treated according to the site of the fistula that was mainly at the junction between the hard and soft palate (Pittsburgh Fistula Classification, type III), and the size of the fistula ranged between $2 \& 10 \mathrm{~mm}$. All of the cases were managed by using either two flap palatoplasty technique which is technically easier and proved to be with low fistula rate postoperative when compared to other techniques $5.1 \%$ for two flap palatoplasty, $6.6 \%$ for Furlow, Wardil-Kilner $12.5 \%$ ) or Double opposing z-plasty technique (Furlow) according to the need for correction of the velopharyngeal dysfunction, as Furlows' technique is reported to improve the velopharyngeal competence by lengthening the soft palate by approaching the soft palate to the posterior pharyngeal wall [9].

The patients were divided into two groups in which, group A was operated upon by one of the previously described techniques (two flap palatoplasty in 9 cases or double opposing z-plasty in one case) with the application of platelet-rich plasma (PRP) injection between the nasal and the 
oral layer. The other group, group B was treated by using one of the previously described techniques (two flap palatoplasty in 9 cases or double opposing z-plasty in one case) without the application of the platelet-rich plasma (PRP).

Palatal fistula has a high rate of recurrence following repair. It was reported by Strujak et al., that fistula rates range as high as $58 \%$ after primary repair, and up to an average recurrence rate of nearly $33 \%$ after fistula repair [10] we thought of improving the wound healing process, and having noted the benefits of PRP offered in other surgical procedures, we decided to study if its application with palatal fistula repair could decrease the recurrence rate.

Clinical application of the PRP in plastic surgery has been described to have several benefits: It is easy and rapid to prepare, low relative cost, minimally invasive, contains abundant amounts of growth factors that promote regeneration of the epithelial tissue and improves angiogenesis thus improves healing of wounds [5], and PRP being autologous without the addition of any other products (the technique we use in our study group) so it is considered a safe method for the patient [11].

In our technique for preparing the PRP, we selected for the double spin method which activates the highest therapeutic concentration of the platelets. First spin is of $1000 \mathrm{rpm}$ for 10 minutes to ensure that the platelets are suspended in the platelet rich plasma. In the second hard spin, we concentrated the platelets at the bottom of the tube into a pellet that was easily re-suspended, by spinning at a high speed of $2500 \mathrm{rpm}$ for a short period of time, for 5 minutes.

Mazzocca et al., compared the double and the single spin techniques and found that the highest platelet concentration was achieved using the double-spin method. The therapeutic concentration of platelets was not achieved by the single-spin method [12], the centrifugation force used in the first spin in our present study was selected according to that used by Perez et al., who stated a high platelet recovery using this force [13].

In the present study, we did not activate the platelets exogenously before its application as this, according to Scherer et al., [14], would de-granulate the platelets prematurely with subsequent growth factors release, which have a very short lifespan. Also, platelets can be naturally activated by endogenous collagen as soon as they are injected and so better results are obtained.
Regarding our statistical results after six months follow-up of the patients of both groups, by clinical examination and by post-operative photography, we found that PRP injection with the repair has good results in accelerating tissue healing and less risk of infection. In our study group, the recurrence rate of the fistula was $25 \%$, while in the control group the recurrence rate of the fistula was $50 \%$, which shows an improvement in the recurrence rate of the palatal fistula, after the surgical repair with $p$-value (0.0508).

It was stated by Cohen et al., [15] and Muzaffar et al., [3] that the rate of fistula occurrence increases according to the number of previous surgical attempts to manage the palatal fistulae. In addition, Gonzalez \& Jiménez [6] claimed that they reduced the recurrence rate of fistula compared to other studies, by using PRP during the closure of recurrent cleft palate fistulae.

The results of our study group were satisfactory, as the rate of recurrence of the palatal fistula was $25 \%$ and in comparison, to the control group which was $50 \%$, and this is due to the beneficial effect of using the PRP with the fistula repair and it is proved that its preparation and application is simple and promotes better healing of scar tissue.

So, we recommend applying PRP during palatal fistula repair despite of the small sample size in our study and we recommend increasing the number of patients in further studies.

\section{REFERENCES}

1- Travis D. Reves and Krischna G.: Patel and Christopher M. Discolo. Adjunctive methods in cleft palate repair and complications. In: Travis T. Tollefson, Steven L. Goudy, ed. Complete cleft care. New York: Thieme Medical, pp. 113-124, 2015.

2- Darren M. Smith, Matthew D. Ford and Joseph E. Losee: Palatoplasty fistulas: Diagnosis, treatment and prevention. In: Richard E. Kirschner, Joseph E Losee, ed. 2 Comprehensive Cleft Care. CRC, pp. 1091-1124., 2016.

3- Muzaffar A.R., Byrd H.S., Rohrich R.J., Johns D.F, LeBlanc D., Beren S.J., et al.: Incidence of cleft palate fistula: An institutional experience with two-stage palatal repair. Plast. Reconstr. Surg., 108: 1515-1518,2001.

4- Denny A.D. and Amm C.A.: Surgical technique for the correction of post palatoplasty fistulae of the hard palate. Plast. Reconstr. Surg., 115: 383-387, 2005.

5- Smith R.G., Gassmann C.J. and Campbell M.S.: Platelet rich plasma: Properties and clinical applications. JLGH, 2: 73-77, 2007.

6- Gonzalez-Sanchez J.G. and Jimenez-Barragan K.: Closure of recurrent cleft palate fistulas with plasma rich in growth factors. Acta Otorhinolaringologica (English edition), 62 (6): 448-453, 2011. 
7- Diah E., Lo L., Yun C., Wang R., Wahyuni L. and Chen Y.: Cleft oronasal fistula: A review of treatment fistula results and a surgical management algorithm proposal. Chang Gung Med. J., 30 (6): 529-537, 2007.

8- Pet M.A., Marty-Grames L., Blount Stahl M., Saltzman B.S., Molter D.W. and Woo A.S.: The Furlow palatoplasty for velopharyngeal dysfunction: Velopharyngeal changes, speech improvements and where they intersect. The Cleft Palate. Craniofacial. J., 52 (1): 12-22, 2015.

9- Bykowski M.R., Naran S., Winger D.G. and Losee J.E.: The rate of oronasal fistula following primary cleft palate surgery: A meta-analysis. The Cleft Palate. Craniofacial. J., 52 (4): 81-87, 2015.

10- Strujak G., Nascimento T., Biron C., Romanowski M., Lima A., Carlini J. et al.: Pedicle tongue flap for palatal fistula closure. J. Craniofac. Surg., 27 (8): 2146-2148, 2016.

11- Sommeling C.E., Heyneman A., Hoeksema H., Verbelen J., Stillaert F. and Monstrey S.: The use of platelet rich plasma in plastic surgery: A systematic review. J. Plast., Reconstr. \& Aesth. Surg., 66 (3): 301-311, 2013.

12- Mazzocca A.D., McCarthy M.B., Chowanie D.M., Cote M.P., Romeo A.A., Bradley J.P., Arciero R.A. and Beitzel K.: Platelet rich plasma differs according to preparation method and human variability. J. Bone. Joint. Surg. Am., 15; 94 (4): 308-316, 2012.

13- Perez A.G., Lana J.F., Rodrigues A.A., Luzo A.C., Belangero W.D. and Santana M.H.: Relevant aspects of centrifugation step in the preparation of platelet rich plasm. ISRN Hematol., 2014.

14- Scherr S.S., Tobalem M., Vigato E., Heit Y., Modarressi A., Hinz B., et al.: Non activated versus thrombin activated platelets on wound healing and fibroblast to myofibroblast differentiation in vivo and in vitro. Plast. Reconstr. Surg., 129: 46e-56e, 2012.

15- Cohen S.R., Kalinowski J., LaRossa D. and Randall P.: Cleft palate fistulas: A multivariate statistical analysis of prevelance, etiology and surgical management. Plast. Reconstr. Surg., 81: 1041-1047, 1991. 